UPRF-92-340

hepth@xxx/9206088

May, 1992

\title{
Loops in the Curvature Matrix Model
}

\author{
G. P. Korchemsky円周 \\ Dipartimento di Fisica, Università di Parma and \\ INFN, Gruppo Collegato di Parma, I-43100 Parma, Italy \\ e-mail: korchemsky@vaxpr.cineca.it
}

\begin{abstract}
Macroscopic loop correlators are investigated in the hermitian one matrix model with the potential perturbed by the higher order curvature term. In the phase of smooth surfaces the model is equivalent to the minimal conformal matter coupled to gravity. The properties of the model in the intermediate phase are similar to that of the discretized bosonic string with the central charge $C>1$. Loop correlators describe the effect of the splitting of the random surfaces. It is shown, that the properties of the surfaces are changed in the intermediate phase because the perturbation modifies the spectrum of the scaling operators.
\end{abstract}

* On leave from the Laboratory of Theoretical Physics, JINR, Dubna, Russia

${ }^{\dagger}$ INFN Fellow 


\section{Introduction}

Computer simulations of the discretized Polyakov's bosonic string indicate [1], that the critical behaviour of the model with the central charge beyond the $C=1$ barrier is governed by the higher order intrinsic curvature terms in the string action. Therefore trying to write the matrix model representation of noncritical strings with the central charge $C>1$ it seems natural to perturb the potential of the matrix model by additional terms taking into account the effects of the intrinsic curvature. One of the possible curvature matrix models was proposed in [2] and was studied in details [3, 使. It was found that the $D=0$ hermitian one matrix model with polynomial potential perturbed by the "higher order curvature" term $\left(\operatorname{Tr} M^{2}\right)^{2}$ has a phase diagram similar to the analogous diagram of the discretized Polyakov's bosonic string. It contains the phase of smooth (Liouville) surfaces, the intermediate phase and the phase of branched polymers. In the first phase the perturbation becomes irrelevant and in the continuum limit the model describes nonunitary minimal $(2,2 m-1)$ conformal matter coupled to $2 D$ gravity [5]. The intermediate phase is the most interesting phase because perturbation becomes relevant here and the string susceptibility exponent takes positive values $\left(\gamma_{s t r}=1 /(m+1)\right)$. The same property was noticed [1] for noncritical strings with the central charge $1<C<4$ and one hopes that investigation of the curvature matrix model may give insight into noncritical strings beyond the $C=1$ barrier. However, to make a correspondence of the curvature matrix model with the continuum theory one has to study the properties of the intermediate phase in more details. The calculation of the correlation functions of microscopic and macroscopic loop operators is the first step in this direction. In the present paper we perform this calculation at the spherical (genus zero) approximation.

The partition function of the $D=0$ hermitian one matrix model perturbed by the higher order curvature term is defined as [3]

$$
\mathrm{e}^{Z(\alpha)}=\int d M \exp \left(-\alpha N \operatorname{Tr} V_{0}(M)+\frac{1}{4} g \alpha^{2}\left(\operatorname{Tr} M^{2}\right)^{2}\right)
$$

where integration is performed over hermitian $N \times N$ matrices $M$. Here, $\log \alpha$ is the "bare" cosmological constant, the constant $g$ couples to the perturbation and even potential $V_{0}(M)$ is given by

$$
V_{0}(M)=\int d x \rho(x) \log (x-M), \quad \rho(x)=\rho(-x)
$$

with function $\rho(x)$ being a parameter of the model. The last term in the exponent of (1.1) takes into account effects of the intrinsic curvature. After expansion of the partition function into sum over random surfaces generated by the potential $V_{0}(M)$, this term opens a possibility for random surfaces to touch each other [2]. In the leading large $N$ (spherical) approximation the factorization property $\left\langle\left(\operatorname{Tr} M^{2}\right)^{2}\right\rangle=\left(\left\langle\operatorname{Tr} M^{2}\right\rangle\right)^{2}+\mathcal{O}\left(N^{0}\right)$ implies that these touchings are measured effectively with the following constant [3]

$$
\bar{g}=-g \alpha\left\langle\frac{1}{N} \operatorname{Tr} M^{2}\right\rangle
$$

where $\langle\ldots\rangle$ represents a connected correlator evaluated with the measure defined in (1.1).

The critical behaviour of the partition function depends on the explicit form of the function $\rho(x)$. To obtain the phase diagram mentioned before this function has to satisfy the following equation for all $c$

$$
c f(c) \equiv \int d x \rho(x)\left(1-\frac{1}{\sqrt{1+\frac{c}{x^{2}}}}\right)=\frac{2}{\alpha_{0}}\left(1+(m+1)\left(1-\frac{c}{c_{0}}\right)^{m}-(m+2)\left(1-\frac{c}{c_{0}}\right)^{m+1}\right)
$$

where $\alpha_{0}$ and $c_{0}$ are arbitrary parameters and $m$ is a positive integer. The calculation of the string susceptibility $\chi=-N^{-2} d^{2} Z(\alpha) / d \alpha^{2} \sim\left(\alpha-\alpha_{c r}\right)^{-\gamma_{s t r}}$ shows [3] that under increasing the touching coupling constant $g$ the model passes through the following phases.

For $g<g_{0}=\frac{16}{\left(\alpha_{0} c_{0}\right)^{2}}$ the model is in the phase of smooth (Liouville) surfaces with the string susceptibility exponent $\gamma_{s t r}=-1 / m,(m=2,3, \ldots)$. The critical values of parameters are

$$
\alpha_{c r}=\alpha_{0}, \quad c_{c r}=c_{0}, \quad \bar{g}\left(\alpha_{c r}\right)=0
$$

where parameter $c<0$ defines the boundary of the cut of one loop correlator, defined below in (1.6) and (1.7). Near the critical point they scale as

$$
\chi \sim c-c_{0} \sim\left(\alpha-\alpha_{0}\right)^{1 / m}, \quad \bar{g}(\alpha) \sim \alpha-\alpha_{0} .
$$


For $g=g_{0}$ the model turns into the intermediate phase with the critical exponent $\gamma_{s t r}=1 /(m+1)$. The critical values of parameters are the same $(1.3)$ as in the previous phase, but their scaling is different

$$
\chi \sim \frac{1}{c-c_{0}} \sim\left(\alpha-\alpha_{0}\right)^{-1 /(m+1)}, \quad \bar{g}(\alpha) \sim\left(\alpha-\alpha_{0}\right)^{m /(m+1)}
$$

For $g>g_{0}$ the touching term dominates in (1.1). The random surfaces are degenerated into branched polymers and the string susceptibility exponent has a maximum value $\gamma_{s t r}=1 / 2$.

Let us calculate the correlation functions of micro- and macroscopic operators in different phases. They are given by expressions like $\operatorname{Tr} M^{2 n}$ for finite and infinite $n$, respectively. One notes, that the same operators appear in the asymptotics of one loop correlator $\langle W(z)\rangle$ for large 2 .

$$
\langle W(z)\rangle=\left\langle\frac{1}{N} \operatorname{Tr} \frac{1}{z-M}\right\rangle=\frac{1}{z}+\frac{1}{z^{3}}\left\langle\frac{1}{N} \operatorname{Tr} M^{2}\right\rangle+\mathcal{O}\left(z^{-5}\right)=\frac{1}{z}+\frac{1}{z^{3}}\left(-\frac{\bar{g}}{g \alpha}\right)+\mathcal{O}\left(z^{-5}\right)
$$

and we will use $\langle W(z)\rangle$ as a generating functional of the loop amplitudes. The general expression for (one-cut) one loop correlator in the model (1.1) is [3]

$$
\langle W(z)\rangle=\frac{\alpha}{2} \int \frac{d x \rho(x)}{z-x}\left(1-\frac{\sqrt{z^{2}+c}}{\sqrt{x^{2}+c}}\right)+\frac{\alpha}{2} \bar{g}\left(z-\sqrt{z^{2}+c}\right)
$$

Here, the explicit form of $c$ and $\bar{g}$ can be found [3] by comparing of this expression with the asymptotic expansion (1.6) and their scaling in different phases near the critical point (1.3) is given by (1.4) and (1.5).

\section{Loop correlators}

In matrix model operators like $\operatorname{Tr} M^{2 n} / l$ with $l=n a^{2 \gamma}$ and $n \rightarrow \infty$ create unmarked holes in the random surface. The boundary loop has length $l$ in units of the lattice spacing $a$ and the exponent $\gamma$ is fixed by the condition $l=$ fixed as $n \rightarrow \infty$. As one will show below, $\gamma$ is related to the string susceptibility exponent as $\gamma=\left|\gamma_{s t r}\right|$. We use the following definition of the macroscopic loop operator

$$
w(l)=\lim _{n \rightarrow \infty, a \rightarrow 0} \operatorname{Tr} M^{2 n} /\left(-c_{0}\right)^{n}, \quad l=n a^{2\left|\gamma_{s t r}\right|}
$$

To reach the continuum limit one introduces scaling variables following [6]. Lattice spacing $a$, renormalized cosmological constant $t$, renormalized string coupling constant $\kappa$ and specific heat $u(t)$ are defined as

$$
\frac{\alpha}{\alpha_{0}}=1+a^{2} t, \quad N a^{2-\gamma_{s t r}}=\kappa^{-1}, \quad \chi=a^{-2 \gamma_{s t r}}(u(t))^{\operatorname{sign}\left(-\gamma_{s t r}\right)}
$$

To find the relation between $u$ and $t$ in different phases (string equation) at the lowest order in $\kappa$ one substitutes (2.1) into (1.4) and (1.5).

To extract macroscopic loop correlators from (1.7) we differentiate the both sides of (11.7) with respect to $\alpha$, take into account the dependence $c=c(\alpha)$ and after some algebra obtain

$$
\frac{d}{d \alpha^{-1}}\left(\alpha^{-1} W(z)\right)=\frac{1}{2 \sqrt{z^{2}+c}} \frac{d c}{d \alpha^{-1}}\left((c f(c))^{\prime}-\frac{1}{2} \bar{g}\right)-\frac{1}{4} \frac{d \bar{g}}{d \alpha^{-1}} \int_{0}^{c} \frac{d x}{\sqrt{z^{2}+x}}
$$

where the function $f(c)$ was defined in (1.2). The derivative $d c / d \alpha$ can be easily found from this expression as follows. One expands the both sides of (2.2) into series in $1 / z$ for large $z$, uses asymptotics (1.6) to evaluate the 1.h.s. as $1 / z+\mathcal{O}\left(z^{-3}\right)$ and then compares coefficients before $1 / z$ to get $d c / d \alpha$. After substitution of this derivative, (2.2) is replaced by

$$
\frac{d}{d \alpha^{-1}}\left(\alpha^{-1} W(z)\right)=\frac{1}{\sqrt{z^{2}+c}}+\frac{1}{4} \frac{d \bar{g}}{d \alpha^{-1}} \int_{0}^{c} d x x \frac{d}{d x} \frac{1}{\sqrt{z^{2}+x}}
$$

\footnotetext{
${ }^{1}$ Loop correlator is odd function of $z$ for even potential
} 
and the comparison of the coefficients before $1 / z^{2 n+1}$ in the large $z$ limit leads to

$$
\frac{d}{d \alpha^{-1}}\left(\alpha^{-1}\left\langle\frac{1}{N} \operatorname{Tr} M^{2 n}\right\rangle\right)=\left(\begin{array}{c}
2 n \\
n
\end{array}\right)\left(-\frac{c}{4}\right)^{n}\left(1+\frac{c}{4} \frac{d \bar{g}}{d \alpha^{-1}} \frac{n}{n+1}\right)
$$

where $\left(\begin{array}{c}2 n \\ n\end{array}\right)=\frac{(2 n) !}{(n !)^{2}}$. For $n=0$ this equation becomes trivial, but for $n=1$ it defines the derivative $\frac{d \bar{g}}{d \alpha^{-1}}$. Notice, that for $\bar{g}=0$ it coincides with an analogous equation in the Kazakov's multicritical model [7]. Now we use equation (2.3) to substitute scaling variables (2.1), to calculate $d \bar{g} / d \alpha$ from (1.4) and (1.5) and to get in the limit $n \rightarrow \infty$ the differential equation for macroscopic loop correlator $\langle w(l)\rangle$. After its integration the macroscopic multiloop correlators $\left\langle w\left(l_{1}\right) \ldots w\left(l_{p}\right)\right\rangle$ can be derived from $\langle w(l)\rangle$ as follows. The identity $\frac{d}{d x} \frac{\delta}{\delta \rho(x)} Z=-\alpha N\left\langle\operatorname{Tr} \frac{1}{x-M}\right\rangle$, following from the definition (1.1) of the model, implies that operator $-\frac{1}{\alpha N} \frac{d}{d x} \frac{\delta}{\delta \rho(x)}$ is a generator of multiloop correlators $\left\langle\prod_{i} \operatorname{Tr} \frac{1}{x_{i}-M}\right\rangle$. The macroscopic loops are found from these correlators as coefficients in the expansion into series in $1 / x_{i}$. Hence, macroscopic loop correlators are generated from the partition function by the operator

$$
w(l)=-\frac{1}{\alpha N} \oint_{\Gamma} \frac{d x}{2 \pi i}\left(-\frac{x^{2}}{c_{0}}\right)^{n} \frac{d}{d x} \frac{\delta}{\delta \rho(x)}, \quad n=l a^{-2\left|\gamma_{s t r}\right|} \rightarrow \infty
$$

where integration over contour $\Gamma$, enclosed singularities of the integrand, was introduced to extract the proper coefficient. In the continuum limit the partition function depends on $\rho(x)$ only through the specific heat $u(t)$, or equivalently through the function $c(t)$, and the derivative in (2.4) acts effectively as $\frac{\delta}{\delta \rho(x)}=\int d t \frac{\delta c(t)}{\delta \rho(x)} \frac{\delta}{\delta c(t)}$. The evaluation of the derivative $\frac{\delta c(t)}{\delta \rho(x)}$ is analogous to that of $\frac{d c}{d \alpha}$ performed before. Namely, one differentiates the both sides of (1.7) with respect of $\rho(x)$. Then, the expression for $\frac{\delta W(z)}{\delta \rho(x)}$ depends on two derivatives $\frac{\delta c}{\delta \rho(x)}$ and $\frac{\delta \bar{g}}{\delta \rho(x)}$. To find them one compares the first two coefficients in the expansion of $\frac{\delta W(z)}{\delta \rho(x)}$ for large $z$ with analogous coefficients fixed by the asymptotics (1.6). The resulting two equations have a solution

$$
\frac{\delta c}{\delta \rho(x)}=-\frac{\frac{\delta}{\delta \rho(x)}\left(c f(c)-\frac{1}{16} g \alpha^{2} c \int_{0}^{c} d y y^{2} f^{\prime}(y)\right)}{\left((c f(c))^{\prime}-\frac{1}{2} \bar{g}\right)\left(1-\frac{1}{16} g(\alpha c)^{2}\right)}
$$

where $c \frac{\delta f(c)}{\delta \rho(x)}=-\left(1+\frac{c}{x^{2}}\right)^{-1 / 2}$ follows from the definition (1.2). After expansion of this expression into series in $1 / z^{2}$ and its substitution into (2.4), the generator of macroscopic loops is given by

$$
w(l)=\frac{2 n}{\alpha N}\left(\begin{array}{c}
2 n \\
n
\end{array}\right) \int d t\left(\frac{c}{4 c_{0}}\right)^{n} \frac{1}{(c f(c))^{\prime}-\frac{1}{2} \bar{g}} \frac{16-\frac{n-1}{n+1} g(\alpha c)^{2}}{16-g(\alpha c)^{2}} \frac{\delta}{\delta c(t)}, \quad n=l a^{-2\left|\gamma_{s t r}\right|} \rightarrow \infty
$$

Let us apply the expressions (2.3) and (2.5) to calculate macroscopic loop correlators in the different phases.

\subsection{Phase of smooth surfaces}

In this phase one has $\gamma_{s t r}=-1 / m$, specific heat (2.1) after substitution into (1.4) obeys the string equation

$$
u^{m}(t)=t
$$

function $c(t)$ scales near the critical value $(1.3)$ as $c / c_{0}=1-a^{2 / m} u(t)$ and $d \bar{g} / d \alpha \sim a^{0}$. Taking into account the explicit form (2.1) of string coupling constant $\kappa^{-1}=N a^{2+1 / m}$ and macroscopic length $l=n a^{2 / m}$, we get in the limit $n \rightarrow \infty$ from (2.3) the following equation for macroscopic loop $w(l)$

$$
\frac{d w(l)}{d t}=-\frac{\kappa^{-1}}{\sqrt{l}} \mathrm{e}^{-l u(t)}
$$

valid up to unessential factor regular for $g<g_{0}$ and its solution is

$$
w(l)=\frac{\kappa^{-1}}{\sqrt{l}} \int_{t}^{\infty} d t^{\prime} \mathrm{e}^{-l u\left(t^{\prime}\right)}
$$


The generator (2.5) is found analogously using (2.1) and (1.4) as

$$
w(l)=-\kappa \sqrt{l} \int d t \dot{u}(t) \mathrm{e}^{-l u(t)} \frac{\delta}{\delta u(t)}
$$

To reproduce (2.7), one acts by this operator on the partition function $Z=-\kappa^{-2}\left(\frac{\partial}{\partial t}\right)^{-2} u(t)$, where negative powers of derivative denote integration. Applying operator (2.8) to (2.7) we find macroscopic two-loop correlator

$$
\left\langle w\left(l_{1}\right) w\left(l_{2}\right)\right\rangle=\sqrt{l_{1} l_{2}} \int_{t}^{\infty} d t^{\prime} \dot{u}\left(t^{\prime}\right) \mathrm{e}^{-\left(l_{1}+l_{2}\right) u\left(t^{\prime}\right)}=\frac{\sqrt{l_{1} l_{2}}}{l_{1}+l_{2}} \mathrm{e}^{-\left(l_{1}+l_{2}\right) u}
$$

and proceeding further we obtain the multiloop correlators

$$
\left\langle w\left(l_{1}\right) \ldots w\left(l_{p}\right)\right\rangle=\kappa^{p-2} \sqrt{l_{1} \cdots l_{p}}\left(-\frac{\partial}{\partial t}\right)^{p-3}\left(\dot{u}(t) \mathrm{e}^{-\left(l_{1}+\cdots+l_{p}\right) u(t)}\right) .
$$

The expressions (2.8) and (2.10) for multiloop correlators in the phase of smooth surfaces coincide with analogous expressions in Liouville theory [8].

\subsection{Intermediate phase}

The string susceptibility exponent has positive value in this phase $\gamma_{s t r}=1 /(m+1)$. The string equation

$$
u^{m+1}(t)=t
$$

and the scaling of parameters: $\frac{c}{c_{0}}=1-u(t) a^{\frac{2}{m+1}}$ and $\frac{d \bar{g}}{d \alpha} \sim u^{-1}(t) a^{-\frac{2}{m+1}}$ follow from (1.5) and (2.1). After substitution of these relations, of the string coupling constant $\kappa^{-1}=N a^{2-1 /(m+1)}$ and macroscopic length $l=n a^{2 /(m+1)}$ into (2.3) one gets the following equation for macroscopic loop

$$
\frac{d w(l)}{d t}=-\frac{\kappa^{-1}}{u \sqrt{l}} \mathrm{e}^{-l u(t)}
$$

The solution has a form

$$
w(l)=\frac{\kappa^{-1}}{\sqrt{l}} \int_{t}^{\infty} d t^{\prime} \frac{1}{u\left(t^{\prime}\right)} \mathrm{e}^{-l u\left(t^{\prime}\right)}
$$

and it differs from (2.7) only by $u$ in the integrand. Calculation of the generator (2.5) in the intermediate phase requires to be careful because for $g=g_{0}$ both numerator and denominator of (2.5) vanish as $n \rightarrow \infty$

$$
w(l)=-\frac{\kappa}{\sqrt{l}} \int d t \dot{u}(t)(1+l u(t)) \mathrm{e}^{-l u(t)} \frac{\delta}{\delta u(t)}
$$

The macroscopic two-loop correlator is found by applying operator (2.14) to (2.13)

$$
\left\langle w\left(l_{1}\right) w\left(l_{2}\right)\right\rangle=\frac{\sqrt{l_{1} l_{2}}}{l_{1}+l_{2}} \mathrm{e}^{-\left(l_{1}+l_{2}\right) u}+\frac{1}{u \sqrt{l_{1} l_{2}}} \mathrm{e}^{-\left(l_{1}+l_{2}\right) u}
$$

This expression differs from two-loop amplitude (2.9) in the Liouville phase only by last term which is singular for $l_{1} \rightarrow 0$ or $l_{2} \rightarrow 0$. Using (2.12) it can be rewritten as

$$
\kappa^{2} u \frac{d}{d t}\left\langle w\left(l_{1}\right)\right\rangle \frac{d}{d t}\left\langle w\left(l_{2}\right)\right\rangle=\left\langle w\left(l_{1}\right) \sigma_{0}\right\rangle \frac{1}{\left\langle\sigma_{0} \sigma_{0}\right\rangle}\left\langle\sigma_{0} w\left(l_{2}\right)\right\rangle
$$

where $\sigma_{0}=-\frac{\partial}{\partial t}$ is the puncture operator. This expression suggests the following interpretation of the last term in (2.15): it describes the effect of the splitting of the random surface into two hemispheres with boundary loops having lengths $l_{1}$ and $l_{2}$. We will show in the next section that the form of random surfaces is changed in the intermediate phase because the touching term changes the properties of the microscopic state associated to the puncture operator. Acting by operator (2.14) on (2.15) one calculates macroscopic multiloop correlators as

$$
\left\langle w\left(l_{1}\right) \ldots w\left(l_{p}\right)\right\rangle=-\kappa^{p-2}\left(\frac{\partial}{\partial t}\right)^{p-3}\left(\dot{u}(t) u^{-2}(t) \pi_{l_{1}}(u(t)) \ldots \pi_{l_{p}}(u(t))\right)
$$

where $\pi_{l}(u)=\frac{u^{2}}{\sqrt{l}} \frac{\partial}{\partial u}\left(\frac{1}{u} \mathrm{e}^{-l u}\right)$. The properties of random surfaces described by this expression are considered in the next section where we analyze the influence of the perturbation on the spectrum of the model.

\footnotetext{
${ }^{2}$ The same result was announced in [4]
} 


\section{From loops to states}

To make the correspondence with the continuum theory one finds the spectrum of scaling operators in both phases. In the phase of smooth (Liouville) surfaces the model describes $(2,2 m-1)$ minimal conformal matter coupled to gravity. As was shown in [8], macroscopic loop correlators (2.10) contain all information about the scaling operators in this phase. It turns out that in the intermediate phase there appears a scaling operator which does not contribute to the macroscopic loops $\left\langle w\left(l_{1}\right) \ldots w\left(l_{p}\right)\right\rangle$ and, as a consequence, the spectrum of the model in this phase differs from the spectrum of Liouville.

The spectrum of scaling operators one finds in a standard way perturbing the string equations (2.6) and (2.11) as

$$
t=u^{1 /\left|\gamma_{s t r}\right|}(t)+\sum_{k \geq 1} t_{k} u^{k}(t)
$$

Here, the term with the (renormalized) constant $t_{k}$ appears after one adds the scaling operator $\sigma_{k}$ to the potential of the matrix model. In the phase of Liouville surfaces the explicit form of $\sigma_{k}$ is well known [7]. Operators $\sigma_{k}$ are given by a sum of local operators $\operatorname{Tr} M^{2 n}$ for $n \leq k$ and their correlation functions are described by the $\mathrm{KdV}$ flow [10]. In the intermediate phase the touching term, being a nonlocal in matrix $M$, becomes relevant and one may expect that some of the scaling operators also may be nonlocal, like $\operatorname{Tr} M^{2 n} \operatorname{Tr} M^{2 k}$. Moreover, we will show in this section that nonlocal scaling operators do appear in the intermediate phase and the redundant "boundary" operator [11] is one of them. [3 $^{3}$

The correlation functions of the scaling operators $\sigma_{k}$ are found by differentiation of the partition function over $t_{k}$. Using the relation $\frac{\partial u}{\partial t_{j}}=-u^{j} \frac{\partial u}{\partial t}$ following from (3.1), the generator of correlators of the scaling operator $\sigma_{k}$ is given by

$$
\sigma_{j}=\frac{\partial}{\partial t_{j}}=-\int d t \dot{u}(t) u^{j}(t) \frac{\delta}{\delta u(t)}
$$

and $\sigma_{0}=-\frac{\partial}{\partial t}=\frac{\partial}{\partial t_{0}}$ is the puncture operator. Scaling operators control the asymptotics of macroscopic loop $w(l)$ for small $l$ when the hole becomes microscopic [8, 9]. The explicit form of the expansion of $w(l)$ one gets from (2.8), (2.14) and (3.2) as

$$
w(l)=\kappa \sum_{n=0}^{\infty} \frac{(-1)^{n}}{n !} l^{n+1 / 2} \sigma_{n}
$$

in the Liouville phase and

$$
w(l)=-\kappa \sum_{n=0}^{\infty} \frac{(-1)^{n}}{n !}(n-1) l^{n-1 / 2} \sigma_{n}
$$

in the intermediate phase. These expressions have two important distinctions. Firstly, the contribution of the puncture operator $\sigma_{0}$ is singular for $l \rightarrow 0$ in the intermediate phase (3.3). It is the puncture operator that leads to singular small $l$ behaviour of the last term in the two loop correlator 2.15) responsible for the splitting of the random surface. Secondly, operator $\sigma_{1}$ disappears from (3.3) and, hence, it does not contribute to the macroscopic loop correlators in the intermediate phase.

The expectation value of the operator $\sigma_{k}$ inserted on the random surface with the boundary length $l$ defines the wave function $\left\langle\sigma_{j}(u) w(l)\right\rangle$ associated to this operator [8, 9]. Using relations (2.7), (2.13) and (3.2) we calculate the wave functions in both phases:

$$
\psi_{j}(u, l)=\left\langle\sigma_{j}(u) w(l)\right\rangle=\kappa^{-1} \sqrt{l} \int_{u}^{\infty} d x x^{j} \mathrm{e}^{-l x}
$$

in the Liouville phase and

$$
\varphi_{j}(u, l)=\left\langle\sigma_{j}(u) w(l)\right\rangle=\frac{\kappa^{-1}}{\sqrt{l}} \int_{u}^{\infty} d x x^{j-2}(1+l x) \mathrm{e}^{-l x}
$$

\footnotetext{
${ }^{3}$ Boundary operator measures the boundary length as $\left\langle\sigma_{B} w(l)\right\rangle=l\langle w(l)\rangle$ and in the matrix model with potential $V(M)$ it is given by 11: $\sigma_{B}=d \operatorname{Tr} V(\lambda M) / d \lambda$ for $\lambda=1$. In the model (1.1) the touching term introduces nonlocal contribution to $\sigma_{B}$
} 
in the intermediate phase. Properties of the wave functions (3.4) were studied in 8. It was shown that (3.4) are closely related to the gravity wave functions of Liouville theory in the minisuperspace approximation. In particular, the wave functions (3.4) satisfy the Wheeler-deWitt constraint 8

$$
\mathcal{H}_{L} \psi_{j}=-j(j+1) \psi_{j}+j(j-1) u^{2} \psi_{j-2}
$$

where $\mathcal{H}_{L}=-\left(l \frac{d}{d l}\right)^{2}+(u l)^{2}+\frac{1}{4}$ is the Liouville hamiltonian in the minisuperspace approximation. The comparison of the matrix model results with that in the continuum theory is based on this equation. In the Liouville theory the wave functions associated to microscopic scaling operators are eigenstates of the Liouville hamiltonian under the additional condition on the relation between the specific heat $u$ and Liouville cosmological constant $\mu$

$$
u=\sqrt{\mu}+\ldots
$$

where dots denote analytical in $\mu$ terms. To reproduce the Liouville wave functions in the phase of smooth surfaces one has to perform the following two transformations [8]. At first, one uses an ambiguity in the definition of the basis of scaling operators $\sigma_{k}$ to choose their linear combinations $\hat{\sigma}_{k}$ whose wave functions $\hat{\psi}_{j}(u, l)=\left\langle\hat{\sigma}_{j}(u) w(l)\right\rangle$ diagonalyze the hamiltonian

$$
\mathcal{H}_{L} \hat{\psi}_{j}(u, l)=-j(j+1) \hat{\psi}_{j}(u, l)
$$

Second, one performs analytical transformation of the "KdV background" $\left\{t_{j}\right\}$ in order to go to the "conformal background" in which the string equation has a form (3.7). The explicit form of these transformation was found in 8 .

It is interesting to note that equation (3.6) is invariant under gauge transformations

$$
\psi_{2 j} \rightarrow \psi_{2 j}+\varepsilon \frac{u^{2 j}}{2 j+1} \psi_{0}, \quad \psi_{2 j+1} \rightarrow \psi_{2 j+1}+\varepsilon \frac{u^{2 j}}{2 j+3} \psi_{1}
$$

for an arbitrary $\varepsilon$ and expression (3.4) is one of the elements of the gauge orbit (3.9). At the same time, the solution of (3.8), having semiclassical asymptotics $\psi_{j}(u, l) \rightarrow 0$ as $l \rightarrow \infty$, is unique 8

$$
\hat{\psi}_{j}(u, l) \equiv\left\langle\hat{\sigma}_{j}(u) w(l)\right\rangle=\kappa^{-1} u^{j+1 / 2} K_{j+1 / 2}(u l)
$$

where $K_{j+1 / 2}(u l)$ is the modified Bessel function and the specific heat obeys the string equation (3.7).

The wave functions of the scaling operators in the intermediate phase are given by (3.5) for $j \geq 0$. One would expect that in the continuum limit the touching term modifies the Liouville hamiltonian and the wave functions (3.5) do not obey simple equations, like (3.6) and (3.8). Nevertheless, one finds after some algebra a remarkable relation for the wave functions (3.5) in the intermediate phase

$$
\mathcal{H}_{L} \varphi_{j}=-j(j-1) \varphi_{j}+j(j-3) u^{2} \varphi_{j-2}
$$

It is invariant under gauge transformations

$$
\varphi_{2 j} \rightarrow \varphi_{2 j}+\varepsilon \frac{u^{2 j}}{2 j-1} \varphi_{0}, \quad \varphi_{2 j+1} \rightarrow \varphi_{2 j+1}+\varepsilon \frac{u^{2 j-2}}{2 j+3} \varphi_{3}
$$

for an arbitrary $\varepsilon$. Equations for the wave functions in both phases, (3.6) and (3.11), look similar. Moreover, it can be easily checked that their solutions are related as

$$
\varphi_{j}(u, l)=\frac{j}{j-1} \psi_{j-1}(u, l), \quad j \geq 2
$$

It is important to recognize, that this relation is not valid for two wave functions: $\varphi_{0}$ and $\varphi_{1}$. As a consequence, the gauge transformations of $\psi_{j}$ and $\varphi_{j}$ are different and the equation (3.13) is not invariant under (3.9) and (3.12). For instance, the expressions (3.4) and (3.5) belong to different gauge orbits and they are related as $\varphi_{j}=\frac{j}{j-1}\left(\psi_{j-1}-\frac{1}{j} u^{j-1} \psi_{0}\right)$, but not 3.13$)$.

The choice of the basis of scaling operators is ambiguous [8]. In the phase of smooth surfaces the ambiguity was fixed by the requirement that the corresponding wave functions $\hat{\psi}_{j}$ have to satisfy the WdW equation (3.8) 
in the conformal background (3.7). In the intermediate phase one has not such a reference point and uses instead the relation (3.13) to fix the basis of scaling operators. Then, equations $(3.13)$ and $(3.10)$ lead to

$$
\mathcal{H}_{L} \hat{\varphi}_{j}(u, l)=-j(j-1) \hat{\varphi}_{j}(u, l)
$$

where the wave function $\hat{\varphi}_{j}$ is equal to the linear combination of $\varphi_{k}$ with $k \leq j$. However, trying to diagonalyze equation (3.11) we meet the following property. Equation (3.11) is not changed if one allows for index $j$ to take negative values and defines the corresponding functions $\varphi_{j}$ using (3.5). In the limit of small length $l$ these functions are singular formally $\varphi_{-j} \stackrel{l \rightarrow 0}{\sim} l^{-1 / 2}, j>0$, but one uses gauge ambiguity (3.12) to transform them as $\varphi_{-j} \rightarrow \hat{\varphi}_{-j}=\varphi_{-j}-\frac{1}{j+1} u^{-j} \varphi_{0}$. The functions $\hat{\varphi}_{-j}$ are regular for small $l$ and they cannot be treated as wave functions of local operators [8, 9]. Only functions $\varphi_{j}$ for $j \geq 0, j \neq 1$ having the asymptotics $\varphi_{j} \stackrel{l \rightarrow 0}{\sim} l^{-|j-1 / 2|}$ are microscopic wave functions. Examining (3.11) for $j=0,1, \ldots$ we obtain that the wave functions $\varphi_{j}$ with $j=0$ and $j \geq 2$ form a linear space under the action of the Liouville hamiltonian. For $j=1$ equation (3.11) is replaced by $\mathcal{H}_{L} \varphi_{1}=-2 u^{2} \varphi_{-1}$ and $\mathcal{H}_{L}$ mixes $\varphi_{1}$ with the functions $\varphi_{2 j+1}$ for negative $j$, since the function $\hat{\varphi}_{-1}$ obeys $\mathcal{H}_{L} \varphi_{-1}=-2 \varphi_{-1}+4 u^{2} \varphi_{-3}$ etc. Note, that in the Liouville phase the functions $\psi_{j}$ for positive and negative $j$ are not mixed under the action of $\mathcal{H}_{L}$ in (3.6). One may try to diagonalyze (3.11) choosing

$$
\hat{\sigma}_{1}=\sigma_{1}-\sum_{j=0}^{\infty} \frac{u^{2 j+2}}{2 j+1} \sigma_{-2 j-1}
$$

and $\hat{\varphi}_{1}=\left\langle\hat{\sigma}_{1} w(l)\right\rangle$, but a careful calculation shows that $\mathcal{H}_{L} \hat{\varphi}_{1}=-\kappa^{-1} \frac{1+l u}{\sqrt{l}} \mathrm{e}^{-l u}$ indicating that the wave function of the scaling operator $\sigma_{1}$ is not a eigenstate of the Liouville hamiltonian.

For $j \geq 2$ the wave functions in different phases are related by (3.13) and using (3.10) we choose the solutions of (3.14) as

$$
\hat{\varphi}_{j}(u, l) \equiv\left\langle\hat{\sigma}_{j}(u) w(l)\right\rangle=\kappa^{-1} u^{j-1 / 2} K_{j-1 / 2}(u l), \quad j \geq 0, j \neq 1
$$

For $j=0$ equation (3.11) implies that $\hat{\varphi}_{0}$ is the zero mode of the hamiltonian $\mathcal{H}_{L}$ and it coincides with the zero mode (3.10) in the Liouville phase. Although the wave functions of the puncture operator are the same in both phases $\left(\hat{\psi}_{0}(u, l)=u \hat{\varphi}_{0}(u, l)\right)$ their contributions to the macroscopic loops are different.

Equations (3.16) and (3.5) define a new basis of scaling operators $\hat{\sigma}_{j}$ connected with the "old" KdV basis $\sigma_{j}$ by the relations

$$
\begin{aligned}
\hat{\sigma}_{j} & =\pi(-1)^{j-1} 2^{j-3 / 2} \sum_{s=0}^{[j / 2]} \frac{2^{-2 s}(j-2 s-1) u^{2 s}}{s !(j-2 s) ! \Gamma(s-j+3 / 2)} \sigma_{j-2 s} \\
\sigma_{j} & =\frac{j !}{j-1} 2^{-j+1 / 2} \sum_{s=0}^{[j / 2]} \frac{(2 j-4 s-1) u^{2 s}}{s ! \Gamma(j-s+1 / 2)} \hat{\sigma}_{j-2 s}
\end{aligned}
$$

Two-point correlators of $\hat{\sigma}_{j}$ can be obtained from (3.17) using the correlators in the KdV basis

$$
\left\langle\sigma_{j} \sigma_{k}\right\rangle=-\frac{\kappa^{-2}}{j+k-1} u^{j+k-1}, \quad\left\langle\hat{\sigma}_{j} \hat{\sigma}_{k}\right\rangle=\kappa^{-2}\left(-\frac{\pi}{2}\right) \frac{u^{2 j-1}}{2 j-1} \delta_{j k}, \quad(j, k \neq 1)
$$

up to terms analytic in $u^{2}$. The scaling operators $\sigma_{1}$ and $\hat{\sigma}_{1}$ do not appear in these relations as it should be. The generator of macroscopic loop (3.3) has a simple form in this basis

$$
w(l)=-\kappa \sum_{j=0, j \neq 1}^{\infty}(-1)^{j}(2 j-1) u^{-j+1 / 2} I_{j-1 / 2}(u l) \hat{\sigma}_{j}
$$

where $I_{j-1 / 2}(u l)$ is the modified Bessel function. Starting from this relation it is possible to explain the origin of the last term in (2.15). For two loop correlator equations (3.19) and (3.16) lead to

$$
\left\langle w\left(l_{1}\right) w\left(l_{2}\right)\right\rangle=-\sum_{j=0, j \neq 1}^{\infty}(-1)^{j}(2 j-1) I_{j-1 / 2}\left(u l_{1}\right) K_{j-1 / 2}\left(u l_{2}\right)
$$


Here, we have a sum over states corresponding to the scaling operators (except of $\hat{\sigma}_{1}$ ) and the $j$-th term is interpreted as a propagator of the $j$-th state. Let us compare (3.20) with an analogous expansion of two-loop correlator in the Liouville phase [8]. After identification of the states (3.13), macroscopic two loop correlators in both phases differ only by the contribution of the $(j=0)$-state corresponding to the puncture operator. In the intermediate phase the contribution of $\hat{\sigma}_{0}$ contains the function $I_{-1 / 2}(u l)$ singular as $l \rightarrow 0$ whereas in the Liouville phase it is replaced by the function $I_{1 / 2}(u l)$ regular for small $l$. Thus, the wave functions of the puncture operator coincide in both phases but their propagators are different. We use the identity $I_{-1 / 2}(z)-I_{1 / 2}(z)=\frac{2}{\pi} K_{1 / 2}(z)=\frac{2}{\pi} K_{-1 / 2}(z)$ to rewrite (3.20) as

$$
\left\langle w\left(l_{1}\right) w\left(l_{2}\right)\right\rangle=\frac{2}{\pi} K_{1 / 2}\left(u l_{1}\right) K_{1 / 2}\left(u l_{2}\right)-\sum_{j=1}^{\infty}(-1)^{j}(2 j-1) I_{j-1 / 2}\left(u l_{1}\right) K_{j-1 / 2}\left(u l_{2}\right)
$$

where the second term is equal to the two loop correlator (2.9) in the Liouville phase [8], but the first term with $K_{1 / 2}(z)=\sqrt{\pi /(2 z)} \mathrm{e}^{-z}$ reproduces the splitting term in (2.15). One transforms the contribution of the puncture operator $\hat{\sigma}_{0}$ to the generator (3.19) in an analogous way and developes the following diagram technics for the calculation of macroscopic loop correlators [8]. The state $\hat{\varphi}_{j}$ associated to the local operator $\hat{\sigma}_{j}$ is represented as an insertion of operator on a hemisphere with boundary length $l$ in fig. 1. The two loop amplitude (3.21) is associated to fig. 2 where the first diagram c orresponds to the splitting term in (2.15). For multiloop correlators (2.16) we get the representation of fig. 3. The correlation functions of scaling operators $\hat{\sigma}_{j}(j \neq 1)$ can be obtained by "sewing" [8] fig. 1 into fig. 3. These rules are not applicable for the operator $\hat{\sigma}_{1}$ because it does not contribute to the macroscopic loops (3.19).

We found that the wave functions of local operators $(3.16)$ and their propagators coincide with analogous expressions in the Liouville phase. However, to identify $\hat{\varphi}_{j}(u, l)$ as microscopic Liouville wave functions one has to identify the cosmological constant in the intermediate phase with the Liouville cosmological constant defined in (3.7). The relation between $\mu$ and the background $t_{0}, \ldots, t_{m-1}$ in the intermediate phase can be found by substituting of (3.7) into (3.1). After this identification we can formulate the properties of the spectrum of scaling operators in the intermediate phase as follows. In the continuum limit, the higher order curvature term being introduced into the Liouville theory changes the propagator of the state associated to the puncture operator and leads to the appearance of a new scaling operator $\hat{\sigma}_{1}$ whose wave function does not obey the WdW constraint (3.14).

The scaling operator $\hat{\sigma}_{1}$ has the following interpretation. In the conformal background (3.7), in the intermediate phase, the cosmological constant couples to the scaling operator $\hat{\sigma}_{m-1}$ and one uses (3.16) to get $\left\langle\hat{\sigma}_{m-1} w(l)\right\rangle=-d\langle w(l)\rangle / d \mu=\kappa^{-1} u^{m-3 / 2} K_{m-3 / 2}(u l)$. Integrating this relation and comparing $\langle w(l)\rangle=$ $2 \kappa^{-1} l^{-1} u^{m-1 / 2} K_{m-1 / 2}(u l)$ with (3.19) we obtain the expectation values of the scaling operators [8]

$$
\left\langle\sigma_{m-1}\right\rangle=\frac{\pi \kappa^{-2}}{(2 m-1)(2 m-3)} u^{2 m-1}, \quad\left\langle\sigma_{m+1}\right\rangle=-\frac{\pi \kappa^{-2}}{(2 m-1)(2 m+1)} u^{2 m+1}
$$

and $\left\langle\sigma_{j}\right\rangle=0$ for $j \neq 1, m-1, m+1$. The expectation value $\left\langle\hat{\sigma}_{1}\right\rangle$ is not fixed in such a way because operator $\hat{\sigma}_{1}$ does not contribute to the macroscopic loop (3.19). One uses instead the relations (3.15), (3.17) and (3.18) to evaluate the correlator $\left\langle\hat{\sigma}_{1} \hat{\sigma}_{m-1}\right\rangle$ ast

$$
\left\langle\hat{\sigma}_{1} \hat{\sigma}_{m-1}\right\rangle=\sqrt{\frac{\pi}{2}} \frac{\kappa^{-2}}{(m-1)(m-2)} u^{m-1}, \quad \text { for even } m
$$

and $\left\langle\hat{\sigma}_{1} \hat{\sigma}_{m-1}\right\rangle=0$ for odd $m$ up to analytical in $u^{2}$ terms. After substitution of (3.7) the integration of $-d\left\langle\hat{\sigma}_{1}\right\rangle / d \mu=\left\langle\hat{\sigma}_{1} \hat{\sigma}_{m-1}\right\rangle$ leads to

$$
\left\langle\hat{\sigma}_{1}\right\rangle=-\frac{\sqrt{2 \pi} \kappa^{-2}}{(m+1)(m-1)(m-2)} u^{m+1}, \quad \text { for even } m
$$

Thus, $\left\langle\hat{\sigma}_{1}\right\rangle \neq 0$ for even $m$ and using (3.22) one may form a vanishing linear combination of $u^{m}\left\langle\hat{\sigma}_{1}\right\rangle, u^{2}\left\langle\hat{\sigma}_{m-1}\right\rangle$ and $\left\langle\hat{\sigma}_{m+1}\right\rangle$ which can be considered as expectation value of the equations of motions in the continuum theory.

\footnotetext{
${ }^{4}$ The correlators of $\hat{\sigma}_{m-1}$ with operators $\sigma_{1}$ and $\sigma_{-2 j-1}$ contain logarithmic singularities $\sim \log u$ for odd $m$ which disappear in the correlator of $\hat{\sigma}_{m-1}$ with the linear combination (3.15). That was the reason for the transition from operator $\sigma_{1}$ to $\hat{\sigma}_{1}$.
} 
As was shown before, in the intermediate phase operators $\hat{\sigma}_{m-1}$ and $\hat{\sigma}_{m+1}$ coincide with analogous operators in the Liouville theory and they can be identified as [8]

$$
\hat{\sigma}_{m+1} \rightarrow-\partial^{2} \phi+\frac{1}{4 \pi \gamma} \hat{R}, \quad u^{2} \hat{\sigma}_{m-1} \rightarrow \frac{\mu}{8 \gamma} \mathrm{e}^{\gamma \phi}, \quad u^{m} \hat{\sigma}_{1} \rightarrow \mu^{m / 2} \mathcal{O}(\phi)
$$

where $\mathcal{O}(\phi)$ is the higher order curvature term. The wave function corresponding to the operator $\hat{\sigma}_{1}$, or equivalently $\mathcal{O}(\phi)$, does not satisfy WdW equation (3.14) and it has logarithmic singularities $\hat{\varphi}_{1} \sim-\kappa^{-1} l^{-1 / 2} \log (l u)$ for small $l$ whereas the Liouville microscopic wave functions (3.4) behave as $l^{-j-1 / 2}$. It strongly suggests [8] that $\mathcal{O}(\phi)$ is essentially nonlocal operator in matter and Liouville fields. As a consequence, there is no microscopic state associated to this operator and the correlation functions, like $\left\langle\hat{\sigma}_{1} \ldots\right\rangle$, have not a diagram representation similar to figs. $1-3$.

\section{Conclusions}

We investigated micro- and macroscopic loop correlators in the hermitian one matrix model with the potential perturbed by the higher order curvature term. Perturbation becomes irrelevant in the phase of smooth surfaces and loop amplitudes coincide with analogous expressions in the $(2,2 m-1)$ minimal conformal field theory coupled to Liouville. In the intermediate phase with the positive string susceptibility exponent $\gamma_{s t r}=1 /(m+1)$ loop correlators (2.15) and (2.16) imply that the perturbation introduces an instability into the formation of the random surfaces. The surfaces start to split into a few smaller surfaces and one suspects that their mean size grows as power of the total area in contrast with logarithmic behaviour in the phase of smooth surfaces. The properties of the random surfaces are changed in the intermediate phase because perturbation modifies the spectrum of the scaling operators. After identification of the Liouville cosmological constant in (3.7) the spectrums in the both phases are very similar. The only difference is that new scaling operator $\hat{\sigma}_{1}$ appears and the propagator of the state associated to the puncture operator is changed in the intermediate phase. The scaling operator $\hat{\sigma}_{1}$, being nonlocal in Liouville and matter fields, does not contribute to the macroscopic loops and its wave function does not satisfy Wheeler-deWitt constraint (3.14). For even $m$ the expectation value of $\hat{\sigma}_{1}$ modifies the Liouville equations of motion by higher order curvature term.

\section{References}

[1] F.David, Nucl. Phys. B257 (1985) 45;

J.Ambjørn, B.Durhuus and J.Fröhlich, Nucl. Phys. B257 (1985) 433;

D.V.Boulatov, V.A.Kazakov, I.K.Kostov and A.A.Migdal, Nucl. Phys. B257 (1985) 641; Phys. Lett. 174B (1986) 87.

[2] S.R.Das, A.Dhar, A.M.Sengupta and S.R.Wadia, Mod. Phys. Lett. A5 (1990) 1041.

[3] G.P.Korchemsky, "Matrix model perturbed by higher order curvature terms", Parma Univ. preprint UPRF92-334 (hepth@xxx/9205014).

[4] L.Alvarez-Gaumé and J.L.F.Barbón, "A proposal for D > 1 strings?", CERN-TH-6464/92 / FTUAM9209 (hepth@xxx/9205010).

[5] A.M.Polyakov, Mod. Phys. Lett. A2 (1987) 893;

V.G.Knizhnik, A.M.Polyakov and A.B.Zamolodchikov, Mod. Phys. Lett. A3 (1988) 819.

[6] E.Brézin and V.Kazakov, Phys. Lett. 236B (1990) 144;

D.J.Gross and A.A.Migdal, Phys. Rev. Lett. 64 (1990) 127;

M.Douglas and S.Shenker, Nucl. Phys. 335B (1990) 635.

[7] V.A.Kazakov, Mod. Phys. Lett. A4 (1989) 2125.

[8] G.Moore, N.Seiberg and M.Staudacher, Nucl. Phys. B362 (1991) 665. 
[9] N.Seiberg, "Notes on quantum Liouville theory and quantum gravity", Rutgers preprint RU-90-29.

[10] T.Banks, M.R.Douglas, N.Seiberg and S.H.Shenker, Phys. Lett. 238B (1990) 279.

[11] E.Martinec, G.Moore and N.Seiberg, Phys. Lett. 263B (1991) 190. 


\section{Figures:}

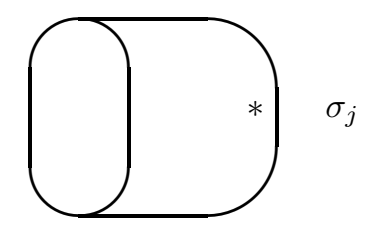

Fig. 1: The wave function associated to the scaling operator $\sigma_{j}$.

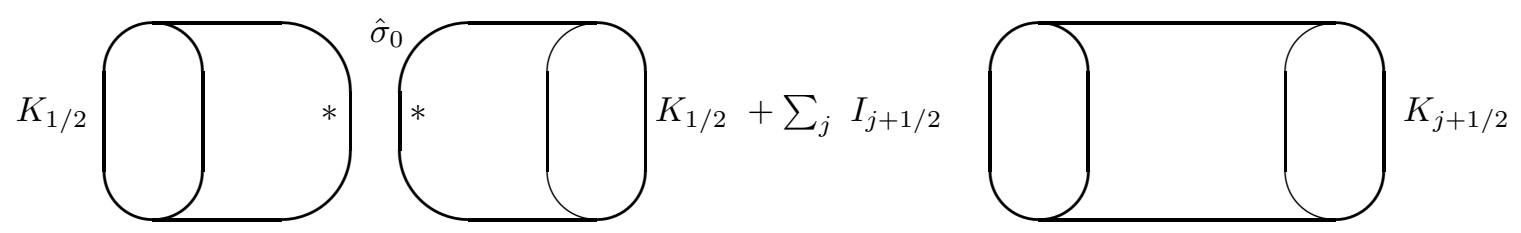

Fig. 2: Macroscopic two-loop amplitude $\left\langle w\left(l_{1}\right) w\left(l_{2}\right)\right\rangle$ in the intermediate phase. The first diagram describes the splitting of the surface.

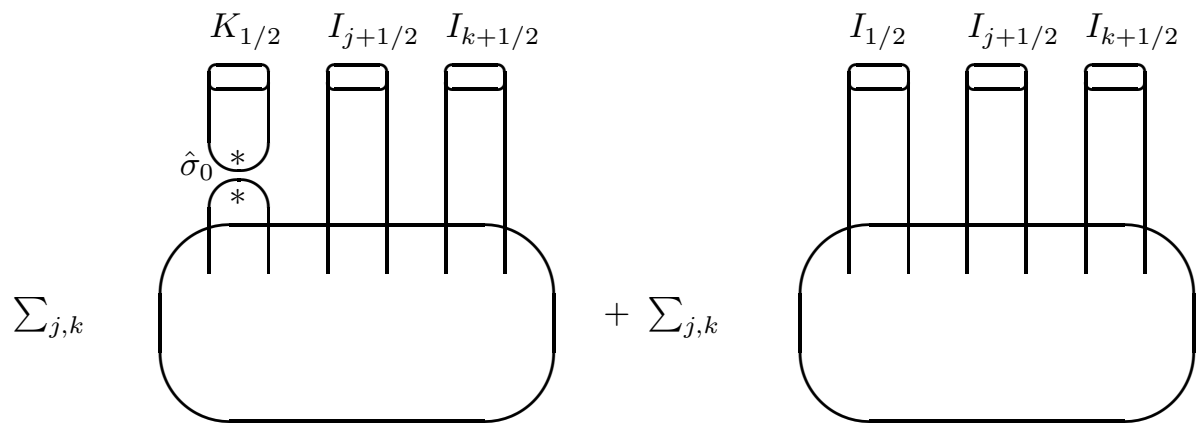

Fig. 3: Some diagrams contributed to the macroscopic multiloop amplitude in the intermediate phase. 